\title{
Aspirin and Colorectal Cancer Prevention and Treatment: Is It for Everyone?
}

\author{
Christopher Coyle $^{1} \cdot$ Fay Helen Cafferty ${ }^{1} \cdot$ Ruth Elizabeth Langley $^{1}$ \\ Published online: 11 February 2016 \\ (C) The Author(s) 2016. This article is published with open access at Springerlink.com
}

\begin{abstract}
There is now a considerable body of data supporting the hypothesis that aspirin could be effective in the prevention and treatment of colorectal cancer, and a number of phase III randomised controlled trials designed to evaluate the role of aspirin in the treatment of colorectal cancer are ongoing. Although generally well tolerated, aspirin can have adverse effects, including dyspepsia and, infrequently, bleeding. To ensure a favourable balance of benefits and risks from aspirin, a more personalised assessment of the advantages and disadvantages is required. Emerging data suggest that tumour PIK3CA mutation status, expression of cyclo-oxygenase-2 and human leukocyte antigen class I, along with certain germline polymorphisms, might all help to identify individuals who stand to gain most. We review both the underpinning evidence and current data, on clinical, molecular and genetic biomarkers for aspirin use in the prevention and treatment of colorectal cancer, and discuss the opportunities for further biomarker research provided by ongoing trials.
\end{abstract}

Keywords Aspirin · Acetylsalicylic acid · NSAID .

Colorectal cancer - Chemoprevention - Secondary prevention . Primary prevention · Adjuvant therapy · Bleeding · PIK3CA . BRAF · Single-nucleotide polymorphism $\cdot$ Human leukocyte antigen

This article is part of the Topical Collection on Personalized Medicine in Colorectal Cancer

Ruth Elizabeth Langley

ruth.langley@ucl.ac.uk

Christopher Coyle

c.coyle@ucl.ac.uk

Fay Helen Cafferty

f.cafferty@ucl.ac.uk

1 MRC Clinical Trials Unit at UCL, Aviation House, 125 Kingsway, London WC2B 6NH, UK

\section{Introduction}

Evidence for the anti-cancer effects of aspirin has emerged from in vitro and animal models, epidemiological studies and randomised data, with the most extensive evidence pertaining to colorectal cancer (CRC). Research suggests that aspirin is effective in primary prevention, reducing the risk of adenomas $[1]$ and CRC $[2 \cdot, 3]$. There is also evidence for a possible role in the treatment of cancer, particularly in the adjuvant setting (preventing recurrence and decreasing the likelihood of metastases after potentially curative therapy) $[4,5 \cdot]$.

The potential benefits of aspirin have to be weighed against the risk of adverse effects, particularly in the primary prevention setting. Upper gastrointestinal symptoms (UGS) are a common concern associated with aspirin use, and can limit adherence, but are usually avoidable. The most undesirable effect of aspirin is an increased bleeding tendency, which can manifest as occult gastrointestinal bleeding, epistaxis or purpura. Serious extra-cranial bleeding is rare (an estimated 3.6 additional events per 10,000 people treated for a year with aspirin [6]), with the vast majority of bleeding episodes resolved without sequelae [7•], and intracranial haemorrhage is rarer still (an estimated 0.8 additional events per 10,000 people treated for a year with aspirin [6]).

Identifying biomarkers or clinical characteristics which predict benefit from aspirin use could lead to a more targeted intervention and protect some individuals from unnecessary treatment and possible side effects. A number of potential clinical, molecular and genetic biomarkers have been evaluated including the following: genes mutated in CRC (PIK3CA and BRAF), molecules proposed to have a role in the mechanism through which aspirin exerts its anti-cancer effects (cyclo-oxygenase (COX) enzymes and human leukocyte antigen (HLA) class I expression), and key genetic polymorphisms that may influence the actions of aspirin.

This review will summarise the current data supporting a personalised approach to aspirin use in relation to CRC and 
highlight the need to discover and validate biomarkers in ongoing trials. The article is structured into four sections: (i) primary prevention of CRC, (ii) treatment of CRC, (iii) safety biomarkers and (iv) other benefit-risk considerations.

\section{Aspirin and Colorectal Cancer Prevention}

The ability of aspirin to prevent colorectal carcinogenesis has been observed in animal models [8-10]. The first clinical evidence emerged in 1988 from a case-control study conducted in Melbourne, Australia, which showed that aspirin reduced the risk of developing CRC [11]. This finding was subsequently corroborated by several other epidemiological studies, with a meta-analysis in 2012 of 30 case-control and cohort studies ( $n=37,519$ CRC cases) showing that aspirin was associated with a lower risk of developing CRC (relative risk (RR) $0.73,95 \%$ confidence interval (CI), 0.67-0.79) [12]. Two large epidemiological studies have recently provided further supporting data. The Association of American Retired Persons Diet and Health study (AARP) included 301,240 adults aged between 50 and 71 years. An estimated $14 \%$ reduction in CRC was observed with daily aspirin use (hazard ratio (HR) 0.86, CI 0.79-0.94) during 10 years of follow-up [13]. A Danish case-control study of 10,280 CRC cases and 102,800 controls showed a reduction in the risk of CRC (odds ratio (OR) 0.73 , CI $0.54-0.99$ ) for those continuously taking aspirin for at least 5 years [14•].

Randomised data substantiate these observations, with a meta-analysis of individual participant data on cancer incidence in randomised trials designed to investigate the effect of aspirin on vascular disease showing that aspirin reduced the 20 -year risk of CRC by $24 \%$ (HR 0.76 , CI $0.63-0.94$ ), improving to $32 \%$ if taken for $\geq 5$ years (HR 0.68 , CI $0.54-0.87$ ) [3]. Similarly, long-term follow-up from the Women's Health Study (WHS), a randomised placebo-controlled trial designed to assess the effects of aspirin (100 mg on alternate days) in the primary prevention of cardiovascular disease, showed that allocation to aspirin reduced the incidence of CRC by $20 \%$ (HR 0.80, CI 0.67-0.97) [2•].

Recommending aspirin for all has been approached with caution due to the difficulties of predicting the chance of benefit, and the risk of toxicity on an individual level and therefore attention, has naturally turned towards individuals at highest risk of CRC who may benefit most.

\section{Aspirin and Colorectal Cancer Prevention: Who Benefits?}

Groups at the highest risk of developing CRC include those with a hereditary CRC syndrome, a history of colorectal adenomas or an inflammatory bowel disease. Aspirin can exacerbate inflammatory bowel disease and is therefore avoided, but there is evidence to support an effect of aspirin in these other high-risk groups.

The most robust evidence exists for Lynch syndrome (hereditary non-polyposis colorectal cancer), the most common inherited CRC syndrome. The CaPP2 (Cancer Prevention Project 2) trial randomly allocated patients with Lynch syndrome to $600 \mathrm{mg}$ daily aspirin or placebo and found a reduction in CRC incidence in those that remained on aspirin for more than 2 years (HR 0.41, CI 0.19-0.86, $p=0.02$ ) [15]. This is in the context of an overall incidence of CRC in the CaPP2 trial population of $5.6 \%(48 / 861)$ over 4.5 years of follow-up, and is likely to represent the group with the highest absolute reduction in risk of CRC. Intriguingly, a sub-analysis of this trial showed that the increase in risk of CRC associated with obesity can be abrogated by aspirin in this population [16•]. Obesity is, thus, a potential predictive biomarker for aspirin benefit, and further research in populations other than Lynch syndrome is warranted. Less evidence is available to support an effect of aspirin in familial adenomatous polyposis (FAP), another inherited CRC syndrome. The CaPP1 trial randomised patients with FAP (prior to preventive surgery), in a $2 \times 2$ factorial design, to $600 \mathrm{mg}$ daily aspirin, resistant starch or placebo. They found a trend towards reduced polyp load in aspirin users; however, this did not reach statistical significance (relative risk 0.77 , CI $0.54-1.10$ ) [17]. The median duration of aspirin use was only 17 months and it is plausible that a treatment effect may have emerged with longer exposure.

Adenomas are precursor lesions for most cases of CRC, and their prevention or regression has been proposed to represent a surrogate marker for CRC risk [18]. A meta-analysis of four randomised controlled trials, including 2967 individuals with previous adenomas or CRC (without an inherited CRC syndrome), found that those allocated to aspirin had a reduced risk of any subsequent adenoma (risk ratio $0.83, \mathrm{CI}$ 0.72-0.96) or advanced adenoma (risk ratio 0.72, CI 0.57 $0.90)$ [1]. This corresponded to a reduction in the absolute risk of any adenoma of $6.7 \%$ (CI 3.2-10.2\%). Further research is needed to confirm whether individuals with a history of adenomas benefit more from aspirin than those without.

Whilst those at highest risk of CRC are most likely to gain from chemoprevention with aspirin, it may be possible to identify those that benefit in lower risk populations. Newly emerging data show that aspirin users with certain singlenucleotide polymorphisms (SNPs) have a reduced risk of developing $\mathrm{CRC}$, and their absence may describe a group who will not benefit. A case-control study of $840 \mathrm{CRC}$ patients and 1686 matched controls examined CRC risk according to expression of the Tallele of rs6983267, which is associated with reduced WNT/ $\beta$-catenin signalling, a major oncogenic pathway in CRC, proposed to be affected by aspirin. They observed that aspirin reduced CRC risk in the cohort as a whole (OR 0.71, CI 0.60-0.85), but the effect was most marked in individuals with a $\mathrm{T}$ allele of rs6983267 (OR 0.83, CI 0.74 
Fig. 1 Studies investigating CRC outcomes according to aspirin use following diagnosis. No summary statistic is presented given the high heterogeneity of studies. Multivariate (adjusted) statistics are presented in all cases. 1 All risk statistics are hazard ratios except the studies by Cardwell [23•] and Rothwell [30], which are odds ratios, and Bastiaannet [26] which is a rate ratio. 2 Studies have an overlapping population, both using UK General Practice Data. 3 Published in abstract form only at time of authorship. 4 Cohort taken from randomised chemotherapy trial and is limited to colon cancer 5 Cohort only matched for CRCspecific mortality analysis. 6 Meta-analysis of cancer outcomes in randomised cardiovascular trials

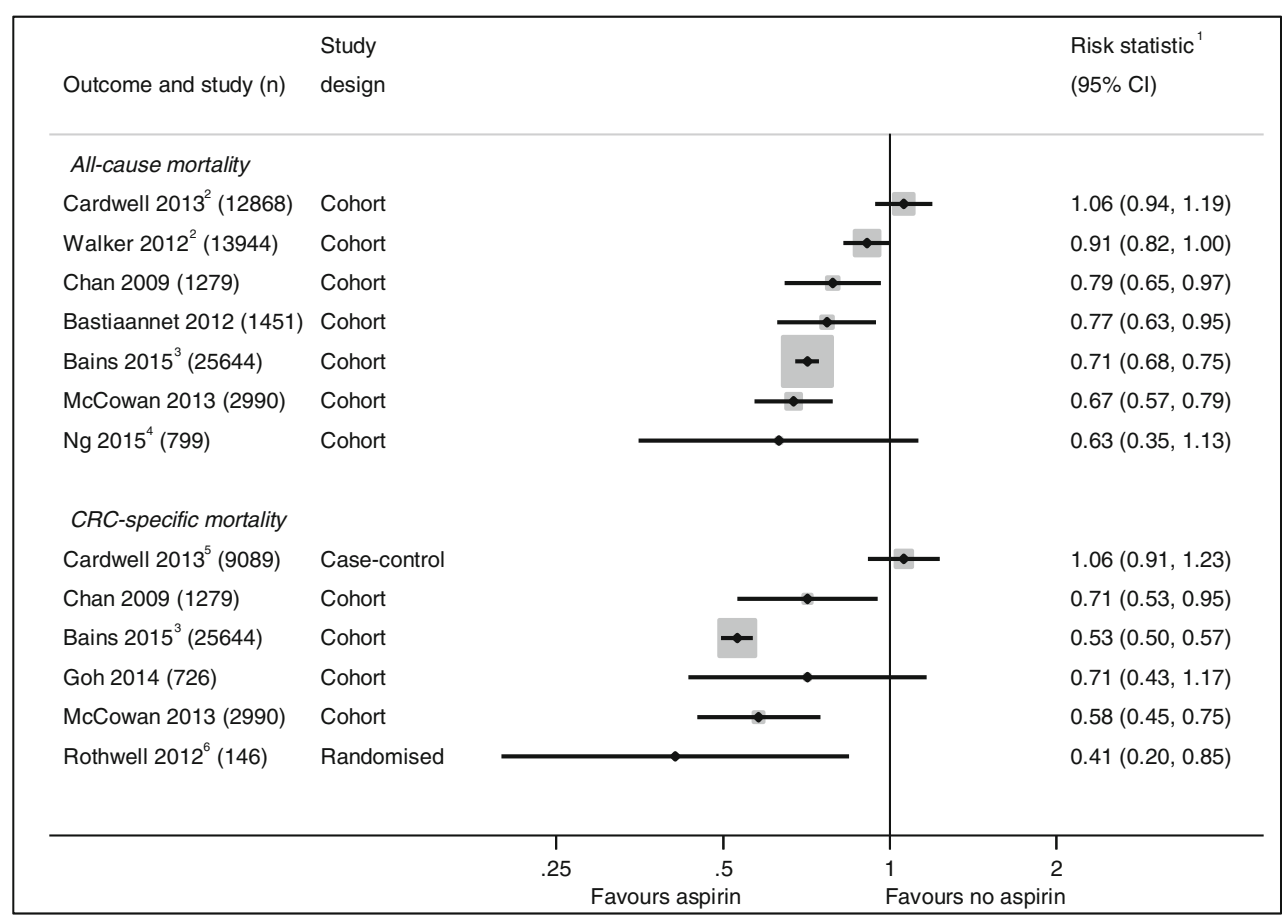

0.94) [19••]. A larger case-control study (8634 cases, 8553 controls) examined the risk of $\mathrm{CRC}$ according to expression of two different SNPs, rs2965667 (located close to the microsomal glutathione S-transferase 1 gene, often upregulated in CRC) and rs16973225 (located close to the interleukin 16 gene, which has been implicated in CRC carcinogenesis) [20••]. It was observed that use of aspirin and/or nonsteroidal anti-inflammatory drugs (NSAIDs) was associated with a reduced risk of CRC amongst individuals with TT genotype of SNP rs2965667 (OR 0.66, CI 0.61-0.70) and the AA genotype of rs16973225 (OR 0.66, CI 0.62-0.71), but not in those with other rarer genotypes. Germline genetic polymorphisms have the potential to identify individuals that benefit from aspirin, as well as those that do not, within populations at lower risk of CRC, and further investigation using existing datasets is required.

\section{Aspirin and Colorectal Cancer Treatment}

In vitro studies show that aspirin inhibits proliferation and induces apoptosis in CRC cell lines [21, 22] suggesting a possible role for aspirin in the treatment of CRC. Figure 1 summarises the results of the clinical studies investigating the effect of aspirin use after a CRC diagnosis. The first epidemiological data emerged from the Nurses' Health Study (NHS) and Health Professionals Follow-up Study (HPFS) showing that regular aspirin use after a diagnosis of CRC is associated with a reduction in CRC deaths (HR 0.71, CI 0.530.95 ) and overall mortality (HR 0.79, CI 0.65-0.97) [24]. This has recently been corroborated by a large cohort of CRC patients from the Cancer Registry of Norway where improvements in overall survival (HR 0.71, CI 0.68-0.75) and CRCspecific survival (HR 0.53, CI 0.50-0.57) were seen with aspirin use after CRC diagnosis [25]. Similar improvements in mortality were observed in data from the Eindhoven Cancer Registry (ECR) (overall survival RR 0.77, CI 0.63-0.95) [26] and in population data collected in Tayside, Scotland (overall mortality HR 0.67 , CI $0.57-0.79$, and CRC-specific mortality HR 0.58, CI 0.45-0.75) [27]. Observational data from the CALGB 89803 trial (which compared two different adjuvant chemotherapy regimens in patients with stage III colon cancer) has also shown a trend towards improved overall survival (HR 0.63, CI 0.35-1.12) and disease-free survival (HR 0.68, CI 0.42-1.11) in those patients using aspirin both during and after chemotherapy [28•]. A study of 13,994 CRC patients from the UK General Practice Research Database found a strong trend towards a reduction in overall mortality; however, this failed to reach significance (HR 0.91, CI 0.82-1.00) [29].

Data on cancer outcomes from randomised trials investigating the effects of aspirin in vascular disease corroborate the trends seen in epidemiological studies. A meta-analysis which included 13,833 individuals who developed CRC in four vascular trials has shown significant reductions in CRC deaths (HR 0.66, CI 0.51-0.85) [3]. Another meta-analysis of five vascular trials has shown that aspirin is associated with a reduction in the risk of having metastases when $\mathrm{CRC}$ is diagnosed (OR 0.36, CI 0.18-0.74) and of subsequently developing them during follow-up when not present at diagnosis (HR 0.26 CI 0.11-0.57) [30]. 
Table 1 Studies examining PIK3CA mutation, aspirin use and colorectal cancer outcomes

\begin{tabular}{|c|c|c|c|c|c|c|c|c|c|c|c|}
\hline \multirow[t]{2}{*}{ Study } & \multirow{2}{*}{$\begin{array}{l}\text { PIK3CA } \\
\text { mutation (\%) }\end{array}$} & \multicolumn{5}{|c|}{ PIK3CA mutant } & \multicolumn{5}{|c|}{ PIK3CA wild type } \\
\hline & & $\begin{array}{l}\text { No } \\
\text { aspirin }\end{array}$ & Aspirin & Outcome & HR & $\begin{array}{l}95 \% \mathrm{CI} \\
p \text { value }\end{array}$ & No aspirin & Aspirin & Outcome & $\mathrm{HR}$ & $\begin{array}{l}95 \% \mathrm{CI} \\
p \text { value }\end{array}$ \\
\hline \multirow{2}{*}{$\begin{array}{l}\text { NHS and } \\
\text { HPFS [36] }\end{array}$} & \multirow[t]{2}{*}{16.7} & \multirow[t]{2}{*}{95} & \multirow[t]{2}{*}{66} & OS & 0.54 & $0.31-0.94 p=0.01$ & \multirow[t]{2}{*}{466} & \multirow[t]{2}{*}{337} & OS & 0.94 & $0.75-1.17 p=0.96$ \\
\hline & & & & CSS & 0.18 & $0.06-0.61 p<0.001$ & & & CSS & 0.96 & $0.69-1.32 p=0.76$ \\
\hline \multirow{2}{*}{$\begin{array}{l}\text { VICTOR } \\
\text { trial [37•] }\end{array}$} & \multirow[t]{2}{*}{11.6} & \multirow[t]{2}{*}{90} & \multirow[t]{2}{*}{14} & OS & 0.29 & $0.04-2.33 p=0.19$ & \multirow[t]{2}{*}{681} & \multirow[t]{2}{*}{111} & OS & 0.95 & $0.56-1.61 p=0.26$ \\
\hline & & & & CSS & 0.11 & $0.001-0.83 p=0.027$ & & & CSS & 0.94 & $0.59-1.49 p=0.79$ \\
\hline \multirow{2}{*}{$\begin{array}{l}\text { MCS and } \\
\text { RMH [38•] }\end{array}$} & \multirow[t]{2}{*}{12.4} & \multirow[t]{2}{*}{136} & \multirow[t]{2}{*}{49} & OS & 0.96 & $0.58-1.57 p=0.86$ & \multirow{2}{*}{\multicolumn{5}{|c|}{ Study of PIK3CA-mutated tumours only }} \\
\hline & & & & CSS & 0.60 & $0.34-1.16 p=0.14$ & & & & & \\
\hline $\operatorname{ECR}^{\mathrm{a}}[33 \bullet \bullet]$ & 15.8 & 73 & 27 & OS & $0.73^{\mathrm{b}}$ & $0.33-1.63 p=0.4$ & 348 & 147 & OS & 0.55 & $0.40-0.75 p<0.001$ \\
\hline
\end{tabular}

Multivariate (adjusted) statistics are presented in all cases

OS overall survival, CSS colorectal cancer-specific survival, RFS recurrence-free survival, NHS Nurses' Health Study, HPFS Health Professionals Follow-up Study, MCS Moffitt Cancer Centre, RMH Royal Melbourne Hospital, ECR Eindhoven Cancer Registry, HR hazard ratio

${ }^{\mathrm{a}}$ Colon cancer only

${ }^{\mathrm{b}}$ Rate ratio

\section{Aspirin and Colorectal Cancer Treatment: Who Benefits?}

Much of the work on potential biomarkers relating to aspirin use as a treatment for CRC has come from three large cohorts (NHS [24], HPFS [24] and ECR [26]). An initial analysis of the NHS/ HPFS dataset reported that the beneficial effects of aspirin on CRC outcomes were restricted to individuals whose tumours overexpressed COX-2 (HR 0.39, CI 0.20-0.76)and not observed for those with weak or absent expression (HR 1.22, CI 0.364.18) [24]. Whilst inhibition of either COX-1 or COX-2 is sufficient to inhibit tumourigenesis in mouse models [31], uncertainty exists about the role of COX enzymes in relation to the anticancer effects of aspirin, particularly given that the daily doses of aspirin used in vascular prevention are not considered sufficient for sustained COX inhibition in systemic tissues [32]. The predictive utility of COX-2 has not been seen in other colorectal studies [33••] or comparative studies in breast cancer [34].

Although the mechanism by which aspirin exerts its anticancer effects remains unknown, one proposed hypothesis was that aspirin, through its anti-platelet effects, could expose circulating tumour cells to immune-mediated destruction by natural killer cells [35] and that this effect would be restricted to tumours with low or absent HLA class I expression. However, analyses of a random sample of colon tumour samples $(n=999)$ from the ECR found that the benefit from aspirin therapy was largely restricted to tumours expressing HLA class I antigens (risk ratio 0.53 , CI $0.38-0.74$ ), and was not seen in those who had lost expression (risk ratio 1.03; CI $0.66-1.61)[33 \cdot \bullet]$. This interesting observation, contrary to the original study hypothesis, requires validation in further datasets. HLA class I expression is seen in about a third of colorectal tumours and so could identify a sizeable group who might benefit from aspirin after a CRC diagnosis.
There has been significant interest in the phosphatidylinositol 3-kinase (PI3KCA) gene as a potential biomarker of aspirin response (Table 1). This follows the publication of data from the NHS/HPFS demonstrating that individuals with PIK3CA mutations taking regular aspirin after a diagnosis of CRC had markedly improved CRC-specific survival (HR 0.18, CI 0.06-0.61) compared to those with wildtype tumours (HR 0.96, CI 0.69-1.32). The same association was observed for overall survival (mutated PIK3CA HR 0.54, CI 0.31-0.94, wild-type PIK3CA HR 0.94, CI 0.75-1.17) [36]. This finding was supported by a small ad hoc analysis of the randomised VICTOR trial, where rofecoxib (a Cox-2 inhibitor) was being evaluated after $\mathrm{CRC}$ resection but the trial was closed early when rofecoxib was withdrawn from the market. Individuals with PIK3CA mutations taking regular aspirin after diagnosis had improved recurrence rates (HR 0.11 , CI 0.001-0.83), whereas those lacking PIK3CA mutation did not (HR 0.92, CI 0.60-1.42), although the number of participants taking aspirin with the mutation was small $(n=14)[37 \bullet]$. However, data from two recent studies has not confirmed the association. In the ECR dataset, the survival benefit associated with aspirin use after a colon cancer diagnosis was seen in those with wild-type PIK3CA tumours (rate ratio 0.55, CI 0.40-0.75), as well as those with PIK3CAmutated tumours where there was a trend towards a survival benefit but this did not reach statistical significance (rate ratio 0.73 , CI $0.33-1.63$ ) [33••]. In addition, in a cohort of patients with PIK3CA-mutated CRCs from the Moffitt Cancer Centre and Royal Melbourne Hospital ( $n=1487)$, no overall survival benefit was observed (HR 0.96, CI 0.58-1.57) and, whilst there was a trend towards a CRC-specific survival benefit, this was not significant (HR 0.60, CI 0.34-1.16) [38•]. PIK3CA mutations only occur in $10-15 \%$ of patients with CRC, whereas the epidemiological data suggest that a greater 
proportion of CRC patients benefit from aspirin use after a CRC diagnosis; therefore, this biomarker needs further investigation, ideally in randomised trials.

A cohort of 1226 patients with a diagnosis of CRC from the NHS and HPFS have also been analysed for BRAF mutation status. The effect of aspirin, after a cancer diagnosis, on cancer-specific and overall survival did not differ according to BRAF mutation status, but may have lacked statistical power. Interestingly, in terms of cancer prevention, aspirin was associated with a lower risk of developing a BRAF wildtype CRC (HR 0.73, CI 0.64-0.83), but not with BRAFmutated CRC (HR 1.03, CI 0.76-1.38) [39•].

The risk of CRC recurrence after potentially curative treatment depends on a number of prognostic factors, which include stage, mode of presentation, microsatellite instability status and whether adjuvant chemotherapy was administered. Any relative improvement in CRC outcomes with aspirin will need to be considered in the context of an individual's absolute risk of recurrence.

\section{Identifying Those at Risk of Toxicity}

Aspirin has been used for over 100 years [40] and has a welldocumented toxicity profile. Standard contraindications to aspirin use include the following: a history of active or recurrent peptic ulceration, active gastrointestinal bleeding, previous intracranial haemorrhage, a haemorrhagic diathesis or a coagulation disorder. Avoiding co-administration of other NSAIDs, anti-coagulants or corticosteroids also reduces the risk of adverse effects [41].

UGS are common side effects associated with aspirin, with one survey reporting $15.4 \%(n=152 / 986)$ of long-term lowdose aspirin users experiencing UGS [42]. A history of gastrooesophageal reflux disease or dyspepsia prior to starting aspirin has been shown to be strongly predictive of UGS on aspirin (OR 17.6, CI 11.52-26.88) [42]. Helicobacter pylori infection has been proposed to be a marker of increased risk of developing dyspepsia and a bleeding gastrointestinal ulcer with aspirin [7•]; however, most data supporting this association relates to non-aspirin NSAID use; and therefore, further data is needed to confirm a relationship with aspirin [43]. The HEAT trial (ISRCTN10134725), examining H. pylori eradication to prevent ulcer-related bleeding and dyspepsia in aspirin users, is ongoing.

The most common cause for concern in relation to aspirin use is the risk of bleeding. Data from six cardiovascular primary prevention RCTs $(n=95,000)$ estimated that aspirin increased the risk of serious bleeding (excluding intracranial haemorrhage) by $0.04 \%$ per year (from 6.6 events per year in 10,000 individuals to 10.2 events [6]). Age has been shown to be a key predictor of bleeding risk with a recent systematic review estimating that the risk of major bleeding increases between three- and fourfold between the ages of 50-54 and 70-74 years [7•]. Intracranial haemorrhage is even rarer with aspirin estimated to increase the risk by less than $0.01 \%$ per year (from 2.7 events in 10,000 individuals treated for a year in the control groups to 3.5 events in the aspirin groups, HR 1.39 , CI 1.08-1.78) in the aforementioned analysis of six cardiovascular RCTs of aspirin. This study also revealed that mean blood pressure is associated with an increased risk of intracranial haemorrhage (rate ratio 2.18, CI 1.65-2.87) [6].

Certain genetic polymorphisms have been proposed as potential biomarkers for NSAID-induced gastrointestinal ulceration and bleeding. A study in a Japanese population $(n=480)$ found that a functional SNP of the COX-1 gene (rs1330344) has been shown to be significantly associated with gastric ulceration (OR 5.80, CI 1.59-21.1) [44]. Additionally, two polymorphisms of CYP2C9 (an enzyme responsible for the metabolism of aspirin) have been found to be significantly associated with bleeding risk in NSAID users [45, 46].

Biomarkers including increasing age, previous dyspepsia and certain SNPs have the potential to identify those at greatest risk of aspirin toxicity. Furthermore, diagnosing and treating conditions like hypertension and $H$. pylori infection could also reduce the change of adverse effects.

\section{Other Benefit-Risk Considerations}

Aspirin is an established treatment for the secondary prevention of cardiovascular disease but is not generally recommended for its primary prevention, however a recommendation for its use in both the prevention of cardiovascular disease and cancer is currently under consideration by the United States Preventive Services Task Force [47]. Little is known about the cardiovascular benefits of aspirin in those with cancer as this is an exclusion criterion in most large cardiovascular trials. CRC and cardiovascular disease have a number of common risk factors, including obesity, high cholesterol and diabetes, and any cardiovascular benefits might add to the rationale for aspirin use in the context of CRC prevention or treatment. Data from cardiovascular trials has additionally suggested that some individuals are resistant to the biological effects of aspirin [48] which also has the potential to limit anti-cancer activity. The existence of aspirin resistance is challenged by the finding that serum thromboxane B2 (a serum marker of platelet activation) is suppressed by aspirin in $99 \%$ of healthy subjects [49]. Other explanations to account for the phenomena include variability in some functional assays of platelet function or undetected poor adherence to aspirin [50]. Fast recovery of platelet function in some individuals might have previously been categorised as resistance, and this might be addressed with twice daily doses [51]. Both the cardiovascular effects of aspirin and the possibility of aspirin resistance could alter the overall risk-benefit profile, and thus require further investigation in existing datasets and ongoing trials. 


\section{Conclusions}

The current data on the benefits and risks of prophylactic aspirin in the general population has recently been reviewed by Cuzick et al. who concluded that aspirin use for greater than 5 years ( $75-$ $325 \mathrm{mg}$ /day), starting between the ages of 55 and 65 , has a favourable benefit-harm profile [52••]. Phase III trials investigating the role of aspirin for cancer prevention in the general population are likely to be challenging due to the length of follow-up and number of participants required. However, in higher risk groups, trials are more feasible. The CaPP3 trial (Cancer Prevention Project 3), examining different doses of aspirin for the prevention of Lynch syndrome cancer (ISRCTN16261285), and the seAFOod (Systematic Evaluation of Aspirin and Fish Oil Bowel Polyp Prevention Trial), examining the effect of aspirin and fish oil in patients at high risk of colorectal adenomas (ISRCTN05926847), are ongoing and the results are awaited.

The role of aspirin in the treatment of CRC is also being evaluated in a number of recruiting phase III trials. AddAspirin (ISRCTN74358648) is a double-blind placebo-controlled trial for patients with colorectal, breast, gastrooesophageal and prostate cancer, investigating the effects of aspirin in the adjuvant setting. Participants are randomised to aspirin $300 \mathrm{mg}$, aspirin $100 \mathrm{mg}$ or placebo for at least 5 years, and it is separately powered for each tumour type to assess the effects of tumour-specific outcomes. Other ongoing trials in this setting include ASCOLT (Aspirin for Dukes C and High Risk Dukes B CRCs, NCT00565708) and ASPIRIN (A Trial of Aspirin on Recurrence and Survival in Elderly Colon Cancer Patients, NCT02301286). There are also three upcoming trials investigating the effects of aspirin in molecularly stratified groups. In the adjuvant setting, ALASCCA is a randomised, double-blind, placebo-controlled phase III trial of aspirin in colorectal patients with mutations in the PI3K signalling pathway (PIK3CA, PIK3R1 or PTEN mutations), and SAKK 41/13 (Swiss Group for Clinical Cancer Research), a double-blind, placebo-controlled randomised trial of adjuvant aspirin treatment in PIK3CA-mutated colon cancer patients (NCT02467582). In the advanced setting, FOCUS4-B (ISRCTN90061546) plans to investigate the role of aspirin in individuals with PIK3CA mutant advanced CRC.

Prior to being considered as an intervention in randomised trials, aspirin has not followed the modern target-driven drug development pathway taken by other anti-cancer agents. Consequently, biomarker discovery and validation is at an early stage. Ideally, predictive biomarkers are co-developed with a drug and validated in phase I and II trials prior to defining the population for phase III trials [53]. However, potential biomarkers for aspirin have mostly emerged from observational studies where there is a risk of confounding and, therefore, it is highly important that they are investigated further in ongoing trials. Research into the mechanisms underlying the anti-cancer effects of aspirin may also reveal new biomarkers. The likelihood of a single biomarker that can identify individuals that will or will not benefit from aspirin is low, and thus, it will be important to investigate patterns of multiple biomarkers to select individuals who will gain from aspirin therapy.

Aspirin is a low-cost, generic agent, available in both resource-poor and resource-rich countries. If it can be shown to be effective, there is the potential for a major impact on the global burden of CRC. Identifying those who are most likely to benefit will be essential to maximising this potential.

\section{Compliance with Ethical Standards}

Conflict of Interest Ruth E. Langley has received financial support through grants from Cancer Research UK and the National Institute for Health Research (NIHR) Health Technology Assessment (HTA) as Chief Investigator of the Add-Aspirin Trial, has received compensation from Bayer and Aspirin Foundation for service on scientific advisory boards, and has received a supply of aspirin and placebo from Bayer Pharmaceuticals AG for the Add-Aspirin Trial.

Human and Animal Rights and Informed Consent This article does not contain any studies with human or animal subjects performed by any of the authors.

Open Access This article is distributed under the terms of the Creative Commons Attribution 4.0 International License (http:// creativecommons.org/licenses/by/4.0/), which permits unrestricted use, distribution, and reproduction in any medium, provided you give appropriate credit to the original author(s) and the source, provide a link to the Creative Commons license, and indicate if changes were made.

\section{References}

Papers of particular interest, published recently, have been highlighted as:

- Of importance

•- Of major importance

1. Cole BF, Logan RF, Halabi S, Benamouzig R, Sandler RS, Grainge MJ, et al. Aspirin for the chemoprevention of colorectal adenomas: meta-analysis of the randomized trials. JNCI J Natl Cancer Inst. 2009;101(4):256-66.

2. Cook NR, Lee IM, Zhang SM, Moorthy MV, Buring JE. Alternateday, low-dose aspirin and cancer risk: long-term observational follow-up of a randomized trial. Ann Intern Med. 2013;159(2): 77-85. This study reports long-term follow-up data from the Women's Health Study. Importantly, it shows that long-term follow-up is necessary to detect the anti-cancer effects of aspirin.

3. Rothwell PM, Wilson M, Elwin CE, Norrving B, Algra A, Warlow $\mathrm{CP}$, et al. Long-term effect of aspirin on colorectal cancer incidence and mortality: 20-year follow-up of five randomised trials. Lancet. 2010;376(9754):1741-50.

4. Langley RE, Burdett S, Tierney JF, Cafferty F, Parmar MK, Venning G. Aspirin and cancer: has aspirin been overlooked as an adjuvant therapy? Br J Cancer. 2011;105(8):1107-13.

5. Langley RE. Clinical evidence for the use of aspirin in the treatment of cancer. Ecancermedicalscience. 2013;(7). This review gives an update on the evidence supporting aspirin use in the adjuvant setting. 
6. Baigent C, Blackwell L, Collins R, Emberson J, Godwin J, Peto R, et al. Aspirin in the primary and secondary prevention of vascular disease: collaborative meta-analysis of individual participant data from randomised trials. Lancet. 2009;373(9678):1849-60.

7. Thorat MA, Cuzick J. Prophylactic use of aspirin: systematic review of harms and approaches to mitigation in the general population. Eur J Epidemiol. 2015;30(1):5-18. This is the most recent systematic review of the harms of aspirin use in the general population, and describes potential strategies to mitigate the risks of adverse effects.

8. Craven PA, DeRubertis FR. Effects of aspirin on 1,2-dimethylhydrazine-induced colonic carcinogenesis. Carcinogenesis. 1992;13(4):541-6.

9. Reddy BS, Rao CV, Rivenson A, Kelloff G. Inhibitory effect of aspirin on azoxymethane-induced colon carcinogenesis in F344 rats. Carcinogenesis. 1993;14(8):1493-7.

10. Barnes CJ, Lee M. Chemoprevention of spontaneous intestinal adenomas in the adenomatous polyposis coli Min mouse model with aspirin. Gastroenterology. 1998;114(5):873-7.

11. Kune GA, Kune S, Watson LF. Colorectal cancer risk, chronic illnesses, operations, and medications: case control results from the Melbourne Colorectal Cancer Study. Cancer Res. 1988;48(15):4399-404.

12. Bosetti C, Rosato V, Gallus S, Cuzick J, La Vecchia C. Aspirin and cancer risk: a quantitative review to 2011. Ann Oncol. 2012;23(6): 1403-15.

13. Ruder EH, Laiyemo AO, Graubard BI, Hollenbeck AR, Schatzkin A, Cross AJ. Non-steroidal anti-inflammatory drugs and colorectal cancer risk in a large, prospective cohort. Am J Gastroenterol. 2011;106(7):1340-50.

14. Friis S, Riis AH, Erichsen R, Baron JA, Sorensen HT. Low-Dose Aspirin or Nonsteroidal Anti-inflammatory Drug Use and Colorectal Cancer Risk: A Population-Based, Case-Control Study. Ann Intern Med. 2015. This is the most recent study supporting the role of aspirin in the prevention of colorectal cancer.

15. Burn J, Gerdes AM, Macrae F, Mecklin JP, Moeslein G, Olschwang $\mathrm{S}$, et al. Long-term effect of aspirin on cancer risk in carriers of hereditary colorectal cancer: an analysis from the CAPP2 randomised controlled trial. Lancet. 2012:378(9809):2081-7.

16. Movahedi M, Bishop DT, Macrae F, Mecklin JP, Moeslein G, Olschwang S, et al. Obesity, Aspirin, and Risk of Colorectal Cancer in Carriers of Hereditary Colorectal Cancer: A Prospective Investigation in the CAPP2 Study. J Clin Oncol. 2015. This subanalysis of the CAPP2 trial shows that obesity may identify those who stand to benefit most from aspirin use in those with Lynch syndrome.

17. Burn J, Bishop DT, Chapman PD, Elliott F, Bertario L, Dunlop $\mathrm{MG}$, et al. A randomized placebo-controlled prevention trial of aspirin and/or resistant starch in young people with familial adenomatous polyposis. Cancer Prev Res (Phila). 2011;4(5):655-65.

18. Kelloff GJ, Schilsky RL, Alberts DS, Day RW, Guyton KZ, Pearce HL, et al. Colorectal adenomas: a prototype for the use of surrogate end points in the development of cancer prevention drugs. Clin Cancer Res. 2004;10(11):3908-18.

19.• Nan H, Morikawa T, Suuriniemi M, Imamura Y, Werner L, Kuchiba A, et al. Aspirin Use, 8q24 Single Nucleotide Polymorphism rs6983267, and Colorectal Cancer According to CTNNB1 Alterations. J Natl Cancer Inst. 2013. This recent study reported that a particular SNP may identify those who benefit from aspirin use for the chemoprevention of colorectal cancer.

20.• Nan H, Hutter CM, Lin Y, Jacobs EJ, Ulrich CM, White E, et al. Association of aspirin and NSAID use with risk of colorectal cancer according to genetic variants. JAMA. 2015;313(11):1133-42. This recent study reports that two further single-nucleotide polymorphisms may identify those who benefit from aspirin use for the chemoprevention of colorectal cancer.
21. Tang X, Sun YJ, Half E, Kuo MT, Sinicrope F. Cyclooxygenase-2 overexpression inhibits death receptor 5 expression and confers resistance to tumor necrosis factor-related apoptosis-inducing ligand-induced apoptosis in human colon cancer cells. Cancer Res. 2002;62(17):4903-8.

22. Elder DJ, Hague A, Hicks DJ, Paraskeva C. Differential growth inhibition by the aspirin metabolite salicylate in human colorectal tumor cell lines: enhanced apoptosis in carcinoma and in vitrotransformed adenoma relative to adenoma relative to adenoma cell lines. Cancer Res. 1996;56(10):2273-6.

23. Cardwell CR, Kunzmann AT, Cantwell MM, Hughes C, Baron JA, Powe DG, et al. Low-dose Aspirin Use After Diagnosis of Colorectal Cancer Does not Increase Survival: a Case-Control Analysis of a Population-Based Cohort. Gastroenterology. 2013. This case-control study utilising UK General Practice data does not show the trend seen in other studies towards an improvement in colorectal cancer outcomes with aspirin use.

24. Chan AT, Ogino S, Fuchs CS. Aspirin use and survival after diagnosis of colorectal cancer. JAMA. 2009;302(6):649-58.

25. Bains S, Mahic M, Cvancarova M, Yaqub S, Dørum ML, Bjørnbeth $\mathrm{BA}$, et al. Impact of aspirin as secondary prevention in an unselected cohort of 25,644 patients with colorectal cancer: a populationbased study. J Clin Oncol 2015;33(suppl; abstr 3504).

26. Bastiaannet E, Sampieri K, Dekkers OM, de Craen AJ, van HerkSukel MP, Lemmens V, et al. Use of aspirin postdiagnosis improves survival for colon cancer patients. Br J Cancer. 2012;106(9):1564 70.

27. McCowan C, Munro AJ, Donnan PT, Steele RJ. Use of aspirin postdiagnosis in a cohort of patients with colorectal cancer and its association with all-cause and colorectal cancer specific mortality. Eur J Cancer. 2013;49(5):1049-57.

28. Ng K, Meyerhardt JA, Chan AT, Sato K, Chan JA, Niedzwiecki D, et al. Aspirin and COX-2 inhibitor use in patients with stage III colon cancer. J Natl Cancer Inst. 2015;107(1):345. This is the most recent study to suggest that aspirin has a role in the adjuvant treatment of cancer.

29. Walker AJ, Grainge MJ, Card TR. Aspirin and other non-steroidal anti-inflammatory drug use and colorectal cancer survival: a cohort study. Br J Cancer. 2012;107(9):1602-7.

30. Rothwell PM, Wilson M, Price JF, Belch JF, Meade TW, Mehta Z. Effect of daily aspirin on risk of cancer metastasis: a study of incident cancers during randomised controlled trials. Lancet. 2012;379(9826):1591-601.

31. Tiano HF, Loftin CD, Akunda J, Lee CA, Spalding J, Sessoms A, et al. Deficiency of either cyclooxygenase (COX)- 1 or COX-2 alters epidermal differentiation and reduces mouse skin tumorigenesis. Cancer Res. 2002;62(12):3395-401.

32. Thun MJ, Jacobs EJ, Patrono C. The role of aspirin in cancer prevention. Nat Rev Clin Oncol. 2012;9(5):259-67.

33.• Reimers MS, Bastiaannet E, Langley RE, van Eijk R, van Vlierberghe RL, Lemmens VE, et al. Expression of HLA Class-I Antigen, Aspirin Use, and Survival After a Diagnosis of Colon Cancer. JAMA Intern Med. 2014. This recent study is the first to investigate the role of HLA class I expression on colorectal cancer outcomes in relation to aspirin use.

34. Holmes MD, Chen WY, Schnitt SJ, Collins L, Colditz GA, Hankinson SE, et al. COX-2 expression predicts worse breast cancer prognosis and does not modify the association with aspirin. Breast Cancer Res Treat. 2011;130(2):657-62.

35. Placke T, Orgel M, Schaller M, Jung G, Rammensee HG, Kopp $\mathrm{HG}$, et al. Platelet-derived MHC class I confers a pseudonormal phenotype to cancer cells that subverts the antitumor reactivity of natural killer immune cells. Cancer Res. 2012;72(2):440-8.

36. Liao X, Lochhead P, Nishihara R, Morikawa T, Kuchiba A, Yamauchi M, et al. Aspirin use, tumor PIK3CA mutation, and colorectal-cancer survival. N Engl J Med. 2012;367(17):1596-606. 
37. Domingo E, Church DN, Sieber O, Ramamoorthy R, Yanagisawa Y, Johnstone E, et al. Evaluation of PIK3CA mutation as a predictor of benefit from nonsteroidal anti-inflammatory drug therapy in colorectal cancer. J Clin Oncol. 2013;31(34):4297-305. This study investigated the role of PIK3CA mutation status on colorectal cancer outcomes and was the first to corroborate the effect seen in the study by Liao et.al.

38. Kothari N, Kim R, Jorissen RN, Desai J, Tie J, Wong HL, et al. Impact of regular aspirin use on overall and cancer-specific survival in patients with colorectal cancer harboring a PIK3CA mutation. Acta Oncol. 2015;54(4):487-92. This is the most recent study to investigate the effects of aspirin in PIK3CA mutant colorectal cancer. No significant improvement in overall survival or cancer-specific survival was observed with aspirin use in those with PIK3CA-mutated colorectal cancer.

39. Nishihara R, Lochhead P, Kuchiba A, Jung S, Yamauchi M, Liao X, et al. Aspirin use and risk of colorectal cancer according to BRAF mutation status. JAMA. 2013;309(24):2563-71. This study examined the role of colorectal cancer BRAF mutation status in relation to aspirin use and found that users had a lower risk of developing a BRAF wild-type tumour.

40. Jack DB. One hundred years of aspirin. Lancet. 1997;350(9075): 437-9.

41. Electronic Medicines Compendium (eMC) SPC aspirin tablets BP 75 mg. 2014. http://www.medicines.org.uk/emc/medicine/26656/ SPC.

42. Cayla G, Collet JP, Silvain J, Thiefin G, Woimant F, Montalescot G. Prevalence and clinical impact of upper gastrointestinal symptoms in subjects treated with low dose aspirin: the UGLA survey. Int $\mathrm{J}$ Cardiol. 2012;156(1):69-75.

43. Fletcher EH, Johnston DE, Fisher CR, Koerner RJ, Newton JL, Gray CS. Systematic review: Helicobacter pylori and the risk of upper gastrointestinal bleeding risk in patients taking aspirin. Aliment Pharmacol Ther. 2010;32(7):831-9.

44. Arisawa T, Tahara T, Shibata T, Nagasaka M, Nakamura M, Kamiya $\mathrm{Y}$, et al. Association between genetic polymorphisms in the cyclooxygenase-1 gene promoter and peptic ulcers in Japan. Int J Mol Med. 2007;20(3):373-8.

45. Pilotto A, Seripa D, Franceschi M, Scarcelli C, Colaizzo D, Grandone E, et al. Genetic susceptibility to nonsteroidal anti- inflammatory drug-related gastroduodenal bleeding: role of cytochrome P450 2C9 polymorphisms. Gastroenterology. 2007;133(2): 465-71.

46. Martinez C, Blanco G, Ladero JM, Garcia-Martin E, Taxonera C, Gamito FG, et al. Genetic predisposition to acute gastrointestinal bleeding after NSAIDs use. Br J Pharmacol. 2004;141(2):205-8.

47. US Preventative Services Task Force. Aspirin to Prevent Cardiovascular Disease and Cancer. 2016. Available from: http:// www.uspreventiveservicestaskforce.org/Page/Document/ UpdateSummaryDraft/aspirin-to-prevent-cardiovascular-diseaseand-cancer? $\mathrm{ds}=1 \& \mathrm{~s}=$ aspirin.

48. Floyd CN, Ferro A. Mechanisms of aspirin resistance. Pharmacol Ther. 2014;141(1):69-78.

49. Santilli F, Rocca B, De Cristofaro R, Lattanzio S, Pietrangelo L, Habib A, et al. Platelet cyclooxygenase inhibition by low-dose aspirin is not reflected consistently by platelet function assays. J Am Coll Cardiol. 2009;53(8):667-77.

50. Dawson J, Quinn T, Rafferty M, Higgins P, Ray G, Lees KR, et al. Aspirin resistance and compliance with therapy. Cardiovasc Ther. 2010.

51. Rocca B, Santilli F, Pitocco D, Mucci L, Petrucci G, Vitacolonna E, et al. The recovery of platelet cyclooxygenase activity explains interindividual variability in responsiveness to low-dose aspirin in patients with and without diabetes. J Thromb Haemost. 2012;10(7): 1220-30.

52.• Cuzick J, Thorat MA, Bosetti C, Brown PH, Burn J, Cook NR, et al. Estimates of benefits and harms of prophylactic use of aspirin in the general population. Ann Oncol. 2014. This article extensively reviews the overall risk-benefit profile of aspirin for the primary prevention of colorectal cancer.

53. Simon R. Advances in clinical trial designs for predictive biomarker discovery and validation. Curr Breast Cancer Rep. 2009;1(4):21621.

54. Goh CH, Leong WQ, Chew MH, Pan YS, Tony LK, Chew L, et al. Post-operative aspirin use and colorectal cancer-specific survival in patients with stage I-III colorectal cancer. Anticancer Res. 2014;34(12):7407-14. This study assessed the effects of aspirin on colorectal cancer specific survival in a predominantly Asian population and found a trend towards benefit with aspirin use. 